\title{
Physician Perceptions of Surveillance Follow-up Colonoscopy in Older Adults
}

\author{
Stephanie T. Le, MD, Brenna R. Lash, MPH, Paul C. Schroy, III, MD, MPH, \\ and Audrey H. Calderwood, MD, MS
}

Background: Few data exist regarding when to stop surveillance colonoscopy among older adults with a history of adenomatous colorectal polyps. Our goal was to understand decision making around surveillance colonoscopy among primary care providers (PCPs) and gastroenterologists.

Methods: We designed a 15-item survey for PCPs and gastroenterologists that evaluated factors important in decision making about surveillance colonoscopy in older adults.

Results: In October 2015, 88 PCPs and 30 gastroenterologists completed the survey. Life expectancy $(40 \%)$, gastroenterology recommendation $(8 \%)$, and patient preference $(12 \%)$ were the most important factors for PCPs. Findings on prior colonoscopy were most important among gastroenterologists. Regardless of specialty, respondents felt that the existing literature on surveillance colonoscopy in older adults is inadequate.

Conclusions: More data surrounding the benefits and risk of surveillance colonoscopy are needed to inform when to stop surveillance colonoscopy among older adults with a positive screening history. (J Am Board Fam Med 2017;30:371-373.)

Keywords: Adenomatous Polyps, Colonoscopy, Colorectal Cancer, Gastroenterology, Primary Health Care, Surveys and Questionnaires

Few data exist regarding when to stop surveillance colonoscopy among older adults with a history of adenomatous colorectal polyps. Continuing surveillance indefinitely could result in unnecessary procedures with little benefit and the potential for harm, particularly given the increased risk of poor preparation, perforation, and cardiopulmonary complications in older adults ${ }^{1}$. This will become an

This article was externally peer reviewed.

Submitted 14 December 2016; revised 18 January 2017; accepted 18 January 2017.

From the Department of Internal Medicine (STL) and the Section of Gastroenterology (BRL, PCS, AHC), Boston Medical Center, Boston, MA; and the Section of Gastroenterology, Dartmouth-Hitchcock Medical Center, Lebanon, NH (AHC).

Funding: AHC was funded by the National Institute of Diabetes and Digestive and Kidney Diseases (grant 1K08DK090150) and a Boston University School of Medicine Department of Medicine Career Investment Award.

Prior presentation: This study was presented in part at Digestive Disease Week, San Diego, CA (May 2016).

Conflict of interest: none declared.

Corresponding author: Audrey H. Calderwood, MD, MS, Section of Gastroenterology, Dartmouth-Hitchcock Medical Center, One Medical Center Drive, Lebanon, NH 03755 (E-mail: Audrey.h.calderwood@hitchcock.org). increasingly important issue as the population ages and more people are found to have polyps. The US Preventive Services Task Force provides a grade C (weak) recommendation that the decision to screen patients between ages 76 to 85 years be individualized, accounting for prior screening and overall health ${ }^{2}$; it provides no recommendation regarding surveillance colonoscopy. ${ }^{3}$ We surveyed primary care providers (PCPs) and gastroenterologists to understand provider decision making around surveillance colonoscopy in adults aged $\geq 75$ years.

\section{Methods}

We designed a 15 -item survey that was administered to trainee and attending PCPs in Internal Medicine, Family Medicine, and Geriatrics at an academic safety net hospital in Boston, Massachusetts. A similar survey was also administered to gastroenterologists, who were members of the Massachusetts Gastroenterology Association and practice in various settings throughout the state. Survey questions addressed the influence of patient age, comorbidities, findings on prior colonosco- 
Table 1. Demographic Characteristics of the Survey Respondents

\begin{tabular}{lcc}
\hline & $\begin{array}{c}\text { Primary Care } \\
\text { Providers* } \\
(\mathrm{n}=88)\end{array}$ & $\begin{array}{c}\text { Gastroenterologists } \\
(\mathrm{n}=30)\end{array}$ \\
\hline Level of training* & & \\
Attending & $36(41)$ & $26(87)$ \\
Fellow & $3(3)$ & $4(13)$ \\
Resident & $47(55)$ & - \\
Time in practice (years) & & $4(13)$ \\
Training & $49(57)$ & $2(7)$ \\
$\leq 5$ & $16(18)$ & $4(13)$ \\
6-10 & $4(5)$ & $8(27)$ \\
$11-20$ & $13(15)$ & $12(40)$ \\
$>20$ & $4(5)$ & - \\
Subspecialty & & - \\
Family & $12(14)$ & - \\
Geriatrics & $18(21)$ & $1(3)$ \\
Internal medicine & $58(65)$ & $10(33)$ \\
Practice setting & & $16(53)$ \\
Community hospital & - & $1(3)$ \\
Ambulatory center & - & \\
Academic hospital & $88(100)$ & - \\
Veterans hospital & - & \\
Other & - & \\
\hline
\end{tabular}

*Data are missing for 2 primary care provider survey respondents who did not provide this information.

pies, and patient preferences; the provider's role in making recommendations; the level of communication between gastroenterologists and PCPs; and the adequacy of existing guidelines. Follow-up rec- ommendations were asked for with regard to 4 patient scenarios that described varying functional status, comorbidities, and colonoscopy findings. Available responses included multiple choice or ranking by priority.

Our primary outcome was the identification of factors important in decision making around surveillance colonoscopy. We performed descriptive statistics and $\chi^{2}$ testing to compare results by specialty and training. This study was considered exempt by the Boston University Medical Center Institutional Review Board.

\section{Results}

A total of 88 PCPs and 30 gastroenterologists completed the survey in October 2015 (Table 1). The majority of PCPs $(75 \%)$ recommended less than half of their older patients undergo surveillance colonoscopy. Life expectancy (40\%), gastroenterology recommendation (28\%), and patient preference $(12 \%)$ were the most important factors in their decisions (Figure 1A). All gastroenterologists reported recommending surveillance colonoscopy in older adults. Findings on prior colonoscopy was ranked as the most important reason to continue surveillance. The majority of gastroenterologists (57\%) ranked comorbidities as the most important factor in recommending against surveillance (Figure 1B). Examination findings ranked much lower among PCPs $(10 \%)$ compared with gastroenterologists $(84 \%)(P<.0001)$.

Figure 1. The most important factors in recommending surveillance colonoscopy among older adults. A (left in blue): Primary care providers (PCPs) ranked the most important factors in influencing decision making around surveillance colonoscopy. B (right in green): Gastroenterologists ranked the most important factors influencing the decision to recommend against surveillance colonoscopy. GI, gastrointestinal.

A

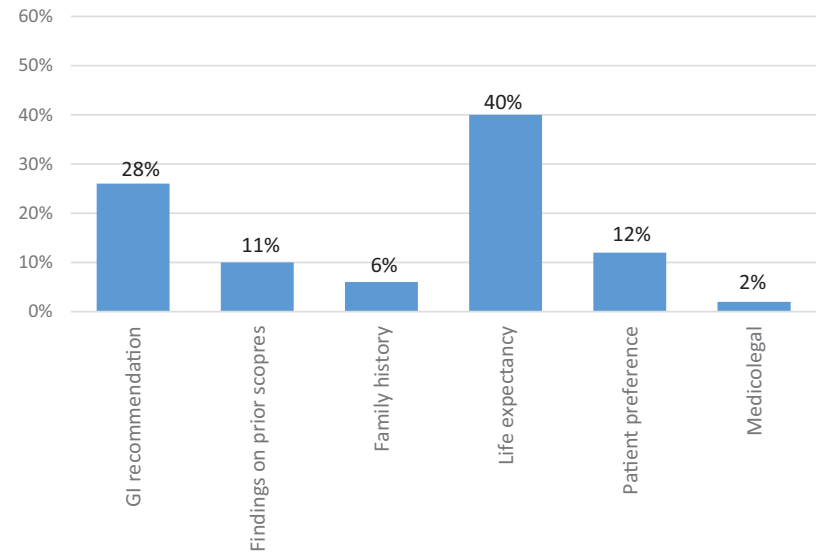

B

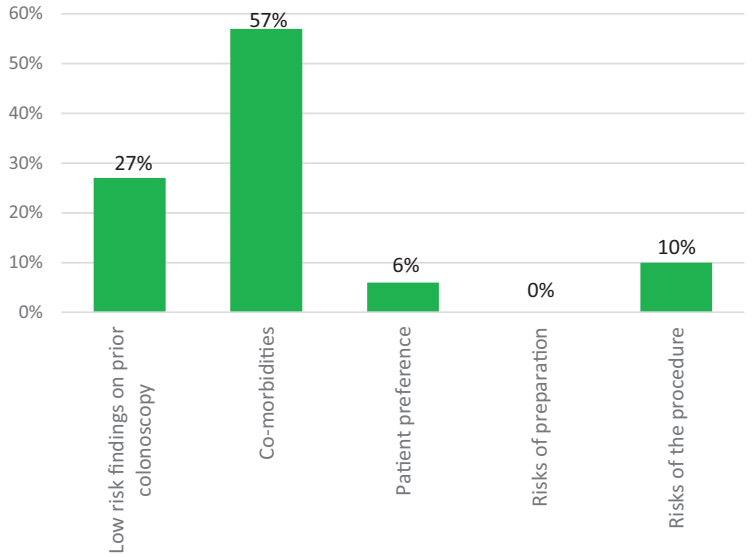


Among healthy older adults, a majority of PCPs (65\%) would follow a gastroenterologist's recommendation regarding colonoscopy; however, this percentage decreased among adults with significant comorbidities (31\%). Similarly, a majority of gastroenterologists $(60 \%)$ would recommend continued surveillance colonoscopy among healthy older adults with high-risk polyps on prior colonoscopies, but would stop surveillance colonoscopy in those with poor function and low-risk lesions (63\%).

Among PCPs, $70 \%$ stated that they have never been contacted by gastroenterology regarding whether to perform surveillance colonoscopy for their patients. Geriatricians (50\%) were more likely than internists $(11 \%)$ and family medicine physicians $(17 \%)$ to consult gastroenterology regarding the need for surveillance colonoscopy in older adult patients $(P=.004)$. Gastroenterologists in private practice were more likely to discuss with PCPs whether to perform surveillance colonoscopy than were gastroenterologists in academic settings (85\% vs $44 \% ; P=.08)$. PCPs felt decisions around surveillance colonoscopy should be between the patient and the PCP (72\%), whereas $56 \%$ of gastroenterologists felt that the primary decision about surveillance colonoscopy should be between the patient and the gastroenterologist.

Most PCPs (76\%) and gastroenterologists $(87 \%)$ felt that the existing literature on surveillance colonoscopy in older adults was inadequate, and that guideline development would be more helpful than provider or patient tools for decision making. These results did not vary based on level of training (resident, fellow, attending) or practice setting.

\section{Discussion}

In this survey of primary care providers and gastroenterologists, we found that physicians value life expectancy and comorbidities as key factors in decision making around surveillance colonoscopy in older adults. In addition, gastroenterologists heavily value abnormal findings on prior colonoscopies. Notably, PCPs and gastroenterologists differ on which provider should have the responsibility for decision making with patients around surveillance colonoscopy. Insufficient communication may occur between PCPs and gastroenterologists regarding this topic, suggesting the opportunity for better communication pathways that enhance joint decision making. More data are needed regarding the relative benefits and risks of surveillance colonoscopy in older adults. ${ }^{4,5}$ Understanding the longterm impact of surveillance colonoscopy would help inform guidelines, promote informed decision making, and help minimize harm to older adults.

To see this article online, please go to: http://jabfm.org/content/ 30/3/371.full.

\section{References}

1. 1. Tran A, Man Ngor E, Wu BU. Surveillance colonoscopy in elderly patients: a retrospective cohort study. JAMA Intern Med 2014;174:1675-82.

2. US Preventive Services Task Force; BibbinsDomingo K, Grossman DC, Curry SJ, et al. Screening for colorectal cancer: US Preventive Services Task Force recommendation statement. JAMA 2016; 315:2564-75.

3. Haggstrom DA, Klabunde KN, Smith JL, Yuan G. Variation in primary care physicians' colorectal cancer screening recommendations by patient age and comorbidity. J Gen Intern Med 2013;28:18-24.

4. Kahi CJ, Azzouz F, Juliar BE, Imperiale TF. Survival of elderly persons undergoing colonoscopy: implications for colorectal cancer screening and surveillance. Gastrointest Endosc 2007;66:544-50.

5. Walter LC, Lindquist K, Nugent $S$, et al. Impact of age and comorbidity on colorectal cancer screening among older veterans. Ann Intern Med 2009;150: 465-73. 